\title{
A!
}

This is an electronic reprint of the original article.

This reprint may differ from the original in pagination and typographic detail.

Kong, Beibei; Yla-Oijala, Pasi; Sihvola, Ari

\section{Surface Integral Equation Method for Soft-and-Hard/DB Boundary Condition}

\section{Published in:}

IEEE Transactions on Antennas and Propagation

DOI:

10.1109/TAP.2020.3030919

Published: 01/05/2021

Document Version

Peer reviewed version

Please cite the original version:

Kong, B., Yla-Oijala, P., \& Sihvola, A. (2021). Surface Integral Equation Method for Soft-and-Hard/DB Boundary Condition. IEEE Transactions on Antennas and Propagation, 69(5), 2790-2797. [9234056].

https://doi.org/10.1109/TAP.2020.3030919

This material is protected by copyright and other intellectual property rights, and duplication or sale of all or part of any of the repository collections is not permitted, except that material may be duplicated by you for your research use or educational purposes in electronic or print form. You must obtain permission for any other use. Electronic or print copies may not be offered, whether for sale or otherwise to anyone who is not an authorised user. 


\title{
Surface Integral Equation Method for Soft-and-Hard/DB Boundary Condition
}

\author{
Beibei Kong, Pasi Ylä-Oijala, and Ari Sihvola, Fellow, IEEE
}

\begin{abstract}
A surface integral equation (SIE) method is developed to analyze electromagnetic scattering by three-dimensional (3-D) objects with Soft-and-Hard/DB (SHDB) boundary condition. The SHDB boundary condition is a generalization of the Soft-and-hard (SH) and DB boundary conditions, which associate the normal and tangential field components on the boundary. In the developed method, the SHDB boundary condition is expressed in vector form which allows combining it with the tangential field integral equations. The obtained equations can be discretized with the standard method of moments (MoM) using the RaoWilton-Glisson (RWG) functions. Different combinations of the integral equations and boundary conditions are derived and their numerical performance are studied and compared. It is demonstrated with numerical experiments that much more stable system is obtained by considering the boundary conditions as extra equations, rather than integrating them to the surface integral equations. The solutions of the proposed non-square integral equation are verified with the physical optics approximations.
\end{abstract}

Index Terms-Electromagnetic scattering, Soft-and-Hard/DB (SHDB) boundary, surface integral equation (SIE)

\section{INTRODUCTION}

$\mathbf{E}$ LECTROMAGNETIC boundary conditions are mathematical models that define the behavior of the electric and magnetic field at the boundary of the region of interest in an electromagnetic problem [1]. Modeling the material interface with a proper set of boundary conditions allows us to avoid considering the fields on the other side of the boundary, thus simplifying the electromagnetic problem and reducing the region of computation. Conventional electromagnetic boundary conditions, including the perfect electric and magnetic conductor (PEC and PMC) boundary conditions and the impedance boundary condition (IBC) [2], are defined in terms of the tangential components of the fields. With the development of artificial electromagnetic materials, nonconventional electromagnetic boundary conditions are introduced to model complex material structures [3]-[5]. The expressions of these non-conventional boundary conditions are more diversified, including tangential fields in specific directions and even normal field components. For example, the Soft-and-hard (SH) boundary condition requires both tangential electric and magnetic fields vanish in one direction [6]. In the DB boundary condition the normal components of the electric and magnetic flux densities vanish on the surface [7], [8].

Recently, the most general linear and local boundary condition, i.e., general boundary condition (GBC), which asso-

The authors are with the Department of Electronics and Nanoengineering, Aalto University, 00076 Espoo, Finland (e-mail: beibei.kong@aalto.fi). ciates the tangential field components with the normal field components, was introduced [9]. As a special case of GBC, the soft-and-hard/DB (SHDB) boundary condition generalizes the SH and DB conditions [10], [11]. The SHDB boundary condition is shown to be one of the most natural and simple boundary conditions expressed in the form of 4-D formalism [12]. Moreover, the SHDB boundary exhibits non-conventional properties to tailor and modify the behavior of electromagnetic waves and has great potential in future engineering applications [10], [11].

The plane wave scattering from an infinite planar SHDB boundary has been investigated analytically [9]-[11]. To be able to analyze the scattering from an arbitrary finite-sized object with SHDB boundary, numerical approaches are needed. However, so far no numerical methods exist for arbitrary SHDB boundaries. This motivates us to develop numerical methods for modeling the interaction of electromagnetic waves with the SHDB boundary. In this paper, we apply a surface integral equation (SIE) method for calculating scattering from arbitrarily shaped 3-D objects with the SHDB boundary. The proposed SIE method enforces the SHDB boundary condition in the weak sense. We express the boundary condition in vector form and then combine it with the tangential field integral equations. The resulting formulations can be discretized using the standard method of moments (MoM) and the Rao-WiltonGlisson (RWG) functions [13]. Alternative ways to combine the field integral equations and the boundary conditions are investigated. The presented results show that combining the SHDB with the field integral equations as a non-square integral equation gives an accurate and stable solution. A physical optics (PO) approximation is developed to analyze reflection properties of planar SHDB surfaces and to verify the solutions of the proposed SIE method.

\section{SOFT-AND-HARD/DB (SHDB) BOUNDARY CONDITION}

\section{A. Definition of $S H D B$}

The Soft-and-Hard/DB (SHDB) condition, introduced in [10], is a generalization of the SH and DB boundary conditions. This condition can be written as [10]

$$
\begin{aligned}
T_{d} \mathbf{n} \cdot c \boldsymbol{B}+T_{s} \mathbf{a}_{t} \cdot \boldsymbol{E} & =0 \\
T_{d} \mathbf{n} \cdot c \boldsymbol{D}-T_{s} \mathbf{a}_{t} \cdot \boldsymbol{H} & =0 .
\end{aligned}
$$

Here $\boldsymbol{D}, \boldsymbol{B}$ are the electric and magnetic flux densities, $\boldsymbol{E}, \boldsymbol{H}$ are the electric and magnetic fields, $T_{d}$ and $T_{s}$ are dimensionless scalar parameters, $\mathbf{n}$ is the unit normal vector pointing outwards the boundary $S, \mathbf{a}_{t}$ is a unit vector tangential with 
$S, c=1 / \sqrt{\varepsilon_{0} \mu_{0}}$, where $\varepsilon_{0}$ and $\mu_{0}$ are the permittivity and permeability of the background medium, respectively.

\section{B. Reflection from $S H D B$}

Consider reflection of an incident time-harmonic plane wave from an infinite planar SHDB boundary. The time factor is $e^{-i \omega t}$. The incident and reflected electric fields are defined as

$$
\boldsymbol{E}^{\mathrm{i}}(\boldsymbol{r})=\boldsymbol{E}^{\mathrm{i}} e^{\left(i k_{0} \mathbf{u}^{\mathrm{i}} \cdot \boldsymbol{r}\right)}, \quad \boldsymbol{E}^{\mathrm{r}}(\boldsymbol{r})=\boldsymbol{E}^{\mathrm{r}} e^{\left(i k_{0} \mathbf{u}^{\mathrm{r}} \cdot \boldsymbol{r}\right)} .
$$

Here $k_{0}=\omega \sqrt{\varepsilon_{0} \mu_{0}}$, unit vectors $\mathbf{u}^{\mathrm{i}}$ and $\mathbf{u}^{\mathrm{r}}$ denote the incident and reflected directions respectively, and satisfy $\mathbf{u}^{\mathrm{r}}=$ $(\overline{\bar{I}}-2 \mathbf{n n}) \cdot \mathbf{u}^{\mathrm{i}}$, in which $\overline{\bar{I}}$ is the unit dyadic.

The reflection dyadics $\overline{\bar{R}}_{E, H}$ can be defined by the relations between the incident and reflected fields, as

$$
\boldsymbol{E}^{\mathrm{r}}=\overline{\bar{R}}_{E} \cdot \boldsymbol{E}^{\mathrm{i}}, \quad \boldsymbol{H}^{\mathrm{r}}=\overline{\bar{R}}_{H} \cdot \boldsymbol{H}^{\mathrm{i}} .
$$

For the SHDB boundary, dyadic $\overline{\bar{R}}_{E}$ reads [1], [9]

$$
\overline{\bar{R}}_{E}=\frac{\mathbf{u}^{\mathrm{r}} \times\left[\mathbf{c}_{2}^{\mathrm{r}} \mathbf{c}_{1}^{\mathrm{i}}-\mathbf{c}_{1}^{\mathrm{r}} \mathbf{c}_{2}^{\mathrm{i}}\right]}{\mathbf{u}^{\mathrm{r}} \cdot \mathbf{c}_{1}^{\mathrm{r}} \times \mathbf{c}_{2}^{\mathrm{r}}}
$$

with

$$
\begin{array}{cc}
\mathbf{c}_{1}^{\mathrm{i}}=T_{d} \mathbf{n} \times \mathbf{u}^{\mathrm{i}}+T_{s} \mathbf{a}_{t}, & \mathbf{c}_{1}^{\mathrm{r}}=T_{d} \mathbf{n} \times \mathbf{u}^{\mathrm{r}}+T_{s} \mathbf{a}_{t} \\
\mathbf{c}_{2}^{\mathrm{i}}=T_{s} \mathbf{u}^{\mathrm{i}} \times \mathbf{a}_{t}+T_{d} \mathbf{n}, & \mathbf{c}_{2}^{\mathrm{r}}=T_{s} \mathbf{u}^{\mathrm{r}} \times \mathbf{a}_{t}+T_{d} \mathbf{n} .
\end{array}
$$

Applying the relations $\boldsymbol{H}^{\mathrm{i}}=\mathbf{u}^{\mathrm{i}} \times \boldsymbol{E}^{\mathrm{i}} / \eta_{0}$ and $\boldsymbol{H}^{\mathrm{r}}=\mathbf{u}^{\mathrm{r}} \times$ $\boldsymbol{E}^{\mathrm{r}} / \eta_{0}$ with $\eta_{0}=\sqrt{\varepsilon_{0} / \mu_{0}}$, we can show that

$$
\overline{\bar{R}}_{H}=-\mathbf{u}^{\mathrm{r}} \times \overline{\bar{R}}_{E} \times \mathbf{u}^{\mathrm{i}}=\overline{\bar{R}}_{E} .
$$

These analytic reflection dyadics are later used to calculate the surface currents for the physical optics (PO) method, which is used to verify the results obtained with the SIE method. In the PO method, the surface of the scatterer is divided into illuminated and shadow regions. The electric and magnetic currents are calculated from the total magnetic and electric field on the illuminated region, as

$$
\boldsymbol{J}^{\mathrm{PO}}=\mathbf{n} \times\left[\boldsymbol{H}^{\mathrm{i}}+\overline{\bar{R}}_{H} \cdot \boldsymbol{H}^{\mathrm{i}}\right], \quad \boldsymbol{M}^{\mathrm{PO}}=-\mathbf{n} \times\left[\boldsymbol{E}^{\mathrm{i}}+\overline{\bar{R}}_{E} \cdot \boldsymbol{E}^{\mathrm{i}}\right],
$$

while on the shadow region, the surface currents are zero. Discretizing the surface with planar elements, the far field radiated by $\boldsymbol{J}^{\mathrm{PO}}$ and $\boldsymbol{M}^{\mathrm{PO}}$ can be calculated in a closed form [14].

\section{Surface Integral Equation Method for SHDB}

\section{A. Surface Integral Formulation}

The main point of this paper is to develop a SIE method for the analysis of electromagnetic scattering by arbitrarily shaped 3-D objects with the SHDB boundary. To this end, let us consider time-harmonic electromagnetic scattering by a closed object with SHDB boundary condition in a linear, homogeneous, and isotropic background medium. For a given incident wave $\left(\boldsymbol{E}^{\mathrm{i}}, \boldsymbol{H}^{\mathrm{i}}\right)$, the tangential field integral equations can be formulated as follows [15]

$$
\left[\begin{array}{cc}
-\gamma_{t} \mathcal{T} & \gamma_{t} \mathcal{K} \\
-\gamma_{t} \mathcal{K} & -\gamma_{t} \mathcal{T}
\end{array}\right]\left[\begin{array}{c}
\eta_{0} \boldsymbol{J} \\
\boldsymbol{M}
\end{array}\right]=\left[\begin{array}{c}
\gamma_{t} \boldsymbol{E}^{\mathrm{i}} \\
\eta_{0} \gamma_{t} \boldsymbol{H}^{\mathrm{i}}
\end{array}\right]
$$

Here $\boldsymbol{J}=\mathbf{n} \times \boldsymbol{H}$ and $\boldsymbol{M}=-\mathbf{n} \times \boldsymbol{E}$ denote the equivalent electric and magnetic surface current densities on the surface $S$, and $\gamma_{t} \boldsymbol{F}$ denotes the tangential component of a vector field $\boldsymbol{F}$ on $S$. The operators $\mathcal{T}$ and $\mathcal{K}$ are defined as

$$
\begin{gathered}
\mathcal{T}\{\boldsymbol{X}\}(\boldsymbol{r})=i k_{0} \int_{S} \boldsymbol{X}\left(\boldsymbol{r}^{\prime}\right) G\left(\boldsymbol{r}, \boldsymbol{r}^{\prime}\right) d S^{\prime}+ \\
\frac{i}{k_{0}} \nabla \int_{S, p . v .} \nabla_{s}^{\prime} \cdot \boldsymbol{X}\left(\boldsymbol{r}^{\prime}\right) G\left(\boldsymbol{r}, \boldsymbol{r}^{\prime}\right) d S^{\prime}-\frac{i}{2 k_{0}} \mathbf{n} \nabla_{s} \cdot \boldsymbol{X}(\boldsymbol{r}) \\
\mathcal{K}\{\boldsymbol{X}\}(\boldsymbol{r})=-\int_{S, p . v .} \boldsymbol{X}\left(\mathbf{r}^{\prime}\right) \times \nabla G\left(\boldsymbol{r}, \boldsymbol{r}^{\prime}\right) d S^{\prime}+\frac{1}{2} \mathbf{n} \times \boldsymbol{X}(\boldsymbol{r})
\end{gathered}
$$

where $G\left(\boldsymbol{r}, \boldsymbol{r}^{\prime}\right)=e^{i k_{0}\left|\boldsymbol{r}-\boldsymbol{r}^{\prime}\right|} / 4 \pi\left|\boldsymbol{r}-\boldsymbol{r}^{\prime}\right|$ is the free-space Green's function of the background with the wavenumber $k_{0}$, $\nabla_{s}$. is the surface divergence, and p.v. stands for the principal value integral.

In order to implement the SHDB boundary condition to the SIEs, the original form of the SHDB needs to be modified and expressed in terms of the equivalent surface electric and magnetic currents. Using well-known identities

$$
\mathbf{n} \cdot \boldsymbol{B}=\frac{1}{i \omega} \nabla_{s} \cdot \boldsymbol{M}, \quad \mathbf{n} \cdot \boldsymbol{D}=\frac{1}{i \omega} \nabla_{s} \cdot \boldsymbol{J},
$$

Eqs. (1) and (2) can be expressed as

$$
\begin{gathered}
i T_{d} \nabla_{s} \cdot \boldsymbol{M}+T_{s} k_{0} \mathbf{n} \times \mathbf{a}_{t} \cdot \boldsymbol{M}=0 \\
i T_{d} \nabla_{s} \cdot \eta_{0} \boldsymbol{J}+T_{s} k_{0} \mathbf{n} \times \mathbf{a}_{t} \cdot \eta_{0} \boldsymbol{J}=0 .
\end{gathered}
$$

The tangential field integral equations are vector equations, while the boundary conditions (13) and (14) are scalar equations. Thus they can not be directly combined to constitute a SIE formulation for the SHDB problem. To that end, we multiply Eqs. (13) and (14) with two orthogonal tangential vectors $\mathbf{a}_{t}$ and $\mathbf{b}_{t}\left(=\mathbf{n} \times \mathbf{a}_{t}\right)$, similarly as in [16] for the GSH surface. This allows us to express the SHDB in vector form as follows

$$
\begin{gathered}
i T_{d} \mathbf{b}_{t}\left(\nabla_{s} \cdot \boldsymbol{M}\right)+T_{s} k_{0} \mathbf{b}_{t}\left(\mathbf{b}_{t} \cdot \boldsymbol{M}\right)=0 \\
i T_{d}\left(\mathbf{n} \times \mathbf{b}_{t}\right)\left(\nabla_{s} \cdot \boldsymbol{M}\right)+T_{s} k_{0}\left(\mathbf{n} \times \mathbf{b}_{t}\right)\left(\mathbf{b}_{t} \cdot \boldsymbol{M}\right)=0 \\
i T_{d} \mathbf{b}_{t}\left(\nabla_{s} \cdot \eta_{0} \boldsymbol{J}\right)+T_{s} k_{0} \mathbf{b}_{t}\left(\mathbf{b}_{t} \cdot \eta_{0} \boldsymbol{J}\right)=0 \\
i T_{d}\left(\mathbf{n} \times \mathbf{b}_{t}\right)\left(\nabla_{s} \cdot \eta_{0} \boldsymbol{J}\right)+T_{s} k_{0}\left(\mathbf{n} \times \mathbf{b}_{t}\right)\left(\mathbf{b}_{t} \cdot \eta_{0} \boldsymbol{J}\right)=0 .
\end{gathered}
$$

Equations (15)-(18) can be combined with the integral equations and discretized with standard MoM using Galerkin's method and RWG functions.

1) Self-Dual Integral Equation (SDIE): Combining Eqs. (15)-(18) with (9), the SIE formulation for the scattering of SHDB boundary can be established as the following self-dual integral equation (SDIE)

$$
\left[\begin{array}{cc}
-\gamma_{t} \mathcal{T}+\mathcal{P} & \gamma_{t} \mathcal{K}-\mathcal{Q} \\
-\gamma_{t} \mathcal{K}+\mathcal{Q} & -\gamma_{t} \mathcal{T}+\mathcal{P}
\end{array}\right]\left[\begin{array}{c}
\eta_{0} \boldsymbol{J} \\
\boldsymbol{M}
\end{array}\right]=\left[\begin{array}{c}
\gamma_{t} \boldsymbol{E}^{\mathrm{i}} \\
\eta_{0} \gamma_{t} \boldsymbol{H}^{\mathrm{i}}
\end{array}\right]
$$

Here the operators $\mathcal{P}$ and $\mathcal{Q}$, according to Eqs. (15)-(18), are defined as

$$
\begin{gathered}
\mathcal{P}\{\boldsymbol{X}\}=i T_{d} \mathbf{b}_{t}\left(\nabla_{s} \cdot \mathbf{X}\right)+T_{s} k_{0} \mathbf{b}_{t}\left(\mathbf{b}_{t} \cdot \boldsymbol{X}\right) \\
\mathcal{Q}\{\boldsymbol{X}\}=i T_{d}\left(\mathbf{n} \times \mathbf{b}_{t}\right)\left(\nabla_{s} \cdot \mathbf{X}\right)+T_{s} k_{0}\left(\mathbf{n} \times \mathbf{b}_{t}\right)\left(\mathbf{b}_{t} \cdot \boldsymbol{X}\right) .
\end{gathered}
$$


Eq. (19) has a similar form as the self-dual formulation for IBC in [17]. Self-dual formulations are also developed for the Mixed IBC [18] and the GSH boundary [16]. However, unlike in these other cases, the SDIE for SHDB does not work well, as will be shown in the numerical examples. Hence, another integral equation formulation is needed.

2) Non-square Integral Equation (NSIE): Another combination of the boundary condition and field integral equations is expressed as

$$
\left[\begin{array}{cc}
-\gamma_{t} \mathcal{T} & \gamma_{t} \mathcal{K} \\
-\gamma_{t} \mathcal{K} & -\gamma_{t} \mathcal{T} \\
\mathcal{P} & -\mathcal{Q} \\
\mathcal{Q} & \mathcal{P}
\end{array}\right]\left[\begin{array}{c}
\eta_{0} \boldsymbol{J} \\
\boldsymbol{M}
\end{array}\right]=\left[\begin{array}{c}
\gamma_{t} \boldsymbol{E}^{\mathrm{i}} \\
\eta_{0} \gamma_{t} \boldsymbol{H}^{\mathrm{i}} \\
0 \\
0
\end{array}\right] .
$$

Compared with SDIE, in Eq. (22) boundary condition and field integral equations are not linearly added with each other, and the discretized matrix of (22) will be a non-square matrix. We call this formulation the non-square integral equation (NSIE). Similar NSIE formulation has been applied previously in [19] for IBC, and for DB and D'B' boundary conditions.

\section{B. Numerical Strategy}

Both SDIE and NSIE are solved numerically with MoM. Using RWG functions and employing the Galerkin's method, the integral equations (19) and (22) are discretized as the following matrix equation, respectively

$$
\begin{gathered}
{\left[\begin{array}{cc}
-\mathbf{T}+\mathbf{P} & \mathbf{K}-\mathbf{Q} \\
-\mathbf{K}+\mathbf{Q} & -\mathbf{T}+\mathbf{P}
\end{array}\right]\left[\begin{array}{l}
\mathbf{x}^{J} \\
\mathbf{x}^{M}
\end{array}\right]=\left[\begin{array}{l}
\mathbf{b}^{E} \\
\mathbf{b}^{H}
\end{array}\right]} \\
{\left[\begin{array}{cc}
-\mathbf{T} & \mathbf{K} \\
-\mathbf{K} & -\mathbf{T} \\
\mathbf{P} & -\mathbf{Q} \\
\mathbf{Q} & \mathbf{P}
\end{array}\right]\left[\begin{array}{l}
\mathbf{x}^{J} \\
\mathbf{x}^{M}
\end{array}\right]=\left[\begin{array}{c}
\mathbf{b}^{E} \\
\mathbf{b}^{H} \\
0 \\
0
\end{array}\right]}
\end{gathered}
$$

with elements

$$
\begin{gathered}
\mathbf{T}_{m n}=\int_{S} \mathbf{g}_{m} \cdot \mathcal{T}\left\{\mathbf{g}_{n}\right\} d S \\
\mathbf{K}_{m n}=\int_{S} \mathbf{g}_{m} \cdot \mathcal{K}\left\{\mathbf{g}_{n}\right\} d S \\
\mathbf{P}_{m n}=i T_{d} \int_{S}\left(\mathbf{g}_{m} \cdot \mathbf{b}_{t}\right)\left(\nabla_{s} \cdot \mathbf{g}_{n}\right) d S+T_{s} k_{0} \int_{S}\left(\mathbf{g}_{m} \cdot \mathbf{b}_{t}\right)\left(\mathbf{b}_{t} \cdot \mathbf{g}_{n}\right) d S \\
\mathbf{Q}_{m n}=i T_{d} \int_{S}\left(\mathbf{g}_{m} \times \mathbf{n} \cdot \mathbf{b}_{t}\right)\left(\nabla_{s} \cdot \mathbf{g}_{n}\right) d S \\
+T_{s} k_{0} \int_{S}\left(\mathbf{g}_{m} \times \mathbf{n} \cdot \mathbf{b}_{t}\right)\left(\mathbf{b}_{t} \cdot \mathbf{g}_{n}\right) d S
\end{gathered}
$$

and excitation vectors

$$
\mathbf{b}_{m}^{E}=\int_{S} \mathbf{g}_{m} \cdot \boldsymbol{E}^{\mathrm{i}} d S, \quad \mathbf{b}_{m}^{H}=\eta_{0} \int_{S} \mathbf{g}_{m} \cdot \boldsymbol{H}^{\mathrm{i}} d S .
$$

Here $\mathbf{g}_{l}(l=m, n)$ is the RWG function associated to the edge $l$. Vectors $\mathbf{x}^{J}$ and $\mathbf{x}^{M}$ include the coefficients of the RWG basis function approximations of $\eta_{0} \boldsymbol{J}$ and $\boldsymbol{M}$, respectively.
We solve the square matrix equation (23) using a direct solver. For the non-square matrix equation (24), we calculate the Pseudo inverse of the matrix to obtain the unknown coefficients of electric and magnetic currents. More specifically, the matrix equation $\mathbf{M x}=\mathbf{b}$ is first transformed as $\left(\mathbf{M}^{H} \mathbf{M}\right) \mathbf{x}=\mathbf{M}^{H} \mathbf{b}$, where ${ }^{H}$ denotes the Hermitian transpose, and then solved using a linear equation solver. The non-square equation is also possible to be solved with the iterative and fast methods [20].

\section{NUMERICAL EXPERIMENTS}

In this section, we investigate numerical solutions of the proposed SIE methods. We first compare SDIE and NSIE with existing methods for the DB and SH boundary conditions and thereafter analyze the numerical performance of these two methods for more general cases. At last, we simulate a large square plate to test the validity and accuracy of the NSIE method.

\section{A. Comparison of SDIE and NSIE}

First, we calculate scattering by a cube with the SHDB boundary and study the numerical performance of SDIE and NSIE. The edge length of the cube is $1 \lambda$, where $\lambda$ is the wavelength of vacuum. The direction of $\mathbf{a}_{t}$ on the surface of the cube is shown in Fig. 1. The cube is illuminated by a plane wave with a frequency of $300 \mathrm{MHz}$. The electric field of the incident plane wave is $x$-polarized and propagating along the $-\mathbf{z}$ direction.

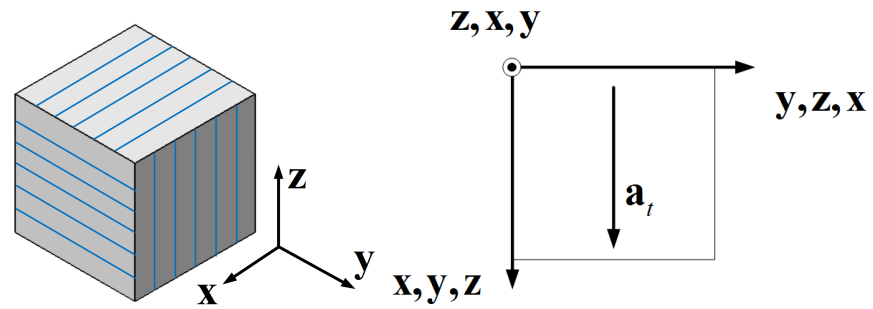

(a)

(b)

Fig. 1. A cube with SHDB surface. (a) The location of the cube in the rectangular coordinate system. The blue lines indicate the direction of $\mathbf{a}_{t}$ on each face of the cube. (b) The direction of $\mathbf{a}_{t}$ on each face of the cube.

1) Limit Cases: From the SHDB boundary condition (13) and (14), it is clear that when $T_{d}=0$, the boundary is the $\mathrm{SH}$ boundary, and when $T_{s}=0$, the boundary is the DB boundary. The bistatic radar cross sections (RCSs) of the SHDB cube are calculated in these limit cases. Meshing the cube with an element size of $\lambda / 10$ gives 2016 edges. For $T_{d}=0, T_{s}=1$, the SDIE has the same form as the SIE formulation in [16], only the coefficients of the boundary term are different. As shown in Fig. 2 (a), the results of SDIE and NSIE agree with the results obtained by the SIE method for SH in [16].

The results for $T_{s}=0, T_{d}=1$ obtained by the proposed two different SIEs are compared with the results of the existing method for DB [21]. As shown in Fig. 2 (b), for the DB case, the results of NSIE agree well with the reference results, while SDIE fails to give an accurate backscattering $\left(\theta=0^{\circ}\right)$ result. 


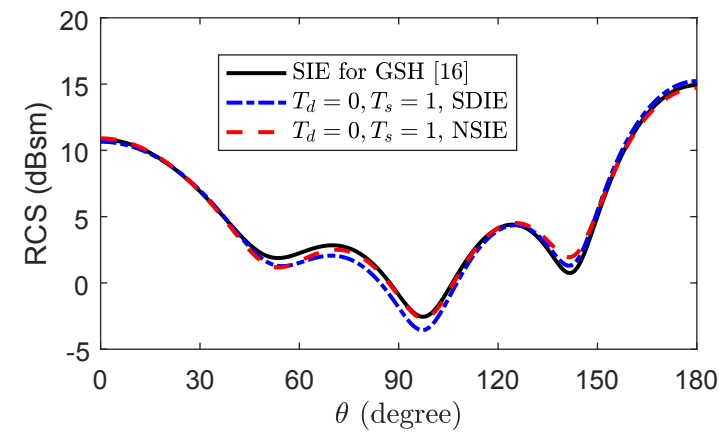

(a)

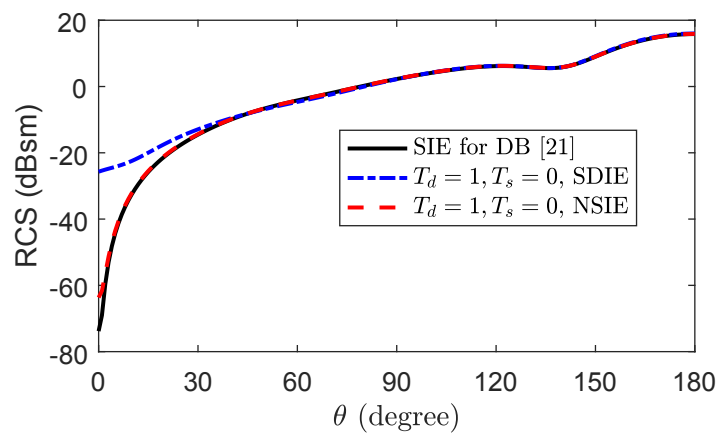

(b)

Fig. 2. Bistatic RCS for the SHDB cube with an edge length of $1 \lambda$ at a frequency of $300 \mathrm{MHz}$. The direction of $\mathbf{a}_{t}$ is shown in Fig. 1. (a) $T_{d}=0$, $T_{s}=1$ (SH surface). (b) $T_{d}=1, T_{s}=0$ (DB surface).

2) Stability of the Formulations: Next we calculate the scattering of the cube with $T_{d}=1$ and $T_{s}=1$, which is a more general case of the SHDB boundary. The bistatic RCS of the cube with two different numbers of edges $N=2016$ and $N=3294$ are shown in Fig. 3. These results indicate that the NSIE gives stable solutions while the solutions SDIE with different mesh densities are totally different.

From Eqs. (13) and (14) we may deduce that parameters $T_{d}=T_{s}=1$ and $T_{d}=T_{s}=0.5$ represent the same boundary. Thus we expect that the SIE method gives the same results when setting $T_{d}=T_{s}=1$ and $T_{d}=T_{s}=0.5$ in (27) and (28). The bistatic RCS of the cube with $N=3294$ and $T_{d}=$ $T_{s}=0.5$ and with $T_{d}=T_{s}=1$ are calculated by the SDIE and NSIE formulations. From Fig. 3, it can be observed that the SDIE fails to give stable results while NSIE is stable with respect to the scaling of the boundary condition parameters.

Letting $T_{s}=\delta$ and $T_{d}=1-\delta$, we calculate the condition number of the matrices for the SHDB cube with $N=3294$ as $\delta$ increases from 0 to 1 . We remind that $\delta=0$ gives a DB boundary and $\delta=1$ an SH boundary. Thus, varying $\delta$ from 0 to 1 contains all possible cases of the SHDB boundary. As can be observed from Fig. 4, SDIE formulation is ill-conditioned except for $\delta>0.9$. This is a probable explanation for the instability of the SDIE results in Fig. $3(\delta=0.5)$. Compared with the SDIE, the condition number of the NSIE formulation is much lower and less oscillating.

From the above results, it can be concluded that NSIE has much better numerical performance than SDIE. The tangential field integral equations define a mapping from the surface currents onto the tangential fields, while SHDB defines a

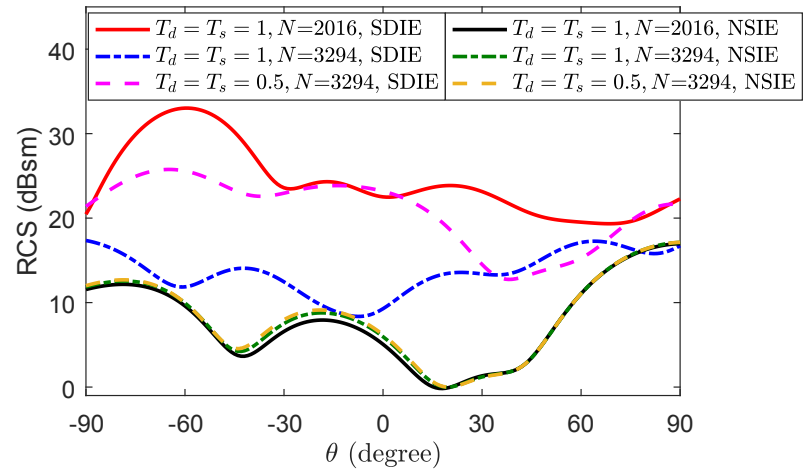

Fig. 3. Bistatic RCS for the SHDB cube with different numbers of edges $N$ and different SHDB coefficients at a frequency of $300 \mathrm{MHz}$. The edge length of the cube is $1 \lambda$ and the direction of $\mathbf{a}_{t}$ is shown in Fig. 1.

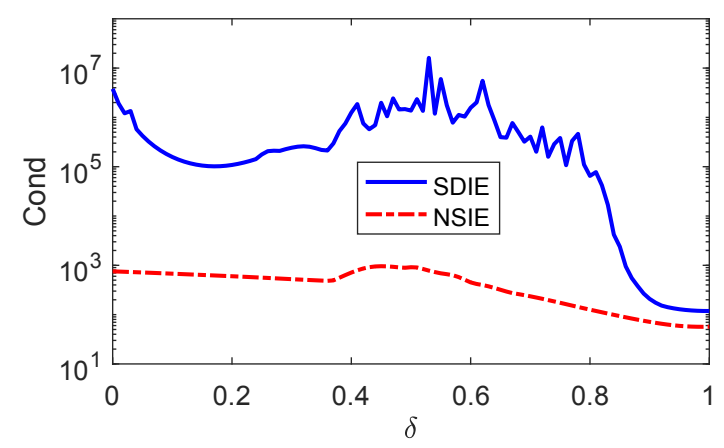

Fig. 4. Condition numbers of the matrices for the SHDB cube with an edge length of $1 \lambda$ and $N=3294$ obtained by SDIE and NSIE versus $\delta$. The operating frequency is $300 \mathrm{MHz}$.

mapping from the currents onto the scalar quantities that can be interpreted as generalized charges. This mismatch of the range spaces of the integral equations and the boundary conditions states that these equations should not be combined, as in the SDIE formulation. This also explains why the results of the SDIE are unstable and more robust solutions are obtained by the NSIE formulation.

\section{B. Scattering from $\mathrm{SHDB}$ plate}

In the following, we calculate scattering from a square plate with the SHDB boundary to verify the results of the NSIE method. To that end we first calculate the co- and cross-polarized components of the reflection dyadic (5) for an infinite planar SHDB plane. The geometry with coordinate system and incident angles is shown in Fig. 5. These reflection coefficients will help us to evaluate and analyze the results of PO and NSIE for a finite-sized plate.

Let $\left(\mathbf{u}^{\mathrm{i}}, \theta^{\mathrm{i}}, \boldsymbol{\varphi}^{\mathrm{i}}\right)$ and $\left(\mathbf{u}^{\mathrm{r}}, \theta^{\mathrm{r}}, \boldsymbol{\varphi}^{\mathrm{r}}\right)$ be two sets of orthogonal unit vectors. For normal incidence,

$$
\varphi^{\mathrm{i}}=\varphi^{\mathrm{r}}
$$

and for oblique incidence, we have

$$
\boldsymbol{\varphi}^{\mathrm{i}}=\mathbf{n} \times \mathbf{u}^{\mathrm{i}} /\left|\mathbf{n} \times \mathbf{u}^{\mathrm{i}}\right|, \quad \boldsymbol{\varphi}^{\mathrm{r}}=\mathbf{n} \times \mathbf{u}^{\mathrm{r}} /\left|\mathbf{n} \times \mathbf{u}^{\mathrm{r}}\right| .
$$

Then $\theta^{\mathrm{i}}$ and $\theta^{\mathrm{r}}$ can be written as

$$
\theta^{\mathrm{i}}=\varphi^{\mathrm{i}} \times \mathbf{u}^{\mathrm{i}}, \quad \theta^{\mathrm{r}}=\boldsymbol{\varphi}^{\mathrm{r}} \times \mathbf{u}^{\mathrm{r}} .
$$




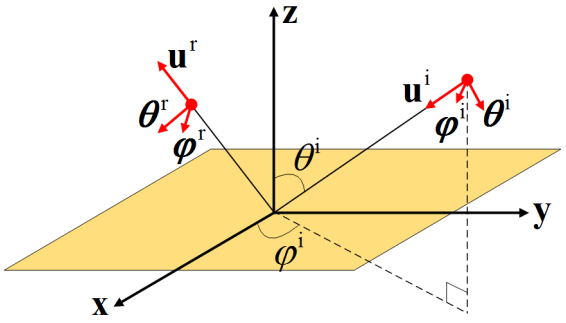

(a)

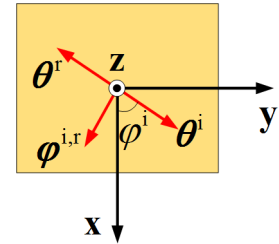

(b)
Fig. 5. Geometry of the reflection problem of an SHDB infinite plane. The direction of $\mathbf{a}_{t}$ is $\mathbf{x}$. (a) Oblique incidence. (b) Normal incidence.

The relations between the incident and reflected fields can be expressed in terms of these two sets of orthogonal unit vectors, as

$$
\boldsymbol{E}^{\mathrm{r}}=\left(\boldsymbol{\varphi}^{\mathrm{r}} \boldsymbol{\varphi}^{\mathrm{i}} R_{\varphi \varphi}+\boldsymbol{\varphi}^{\mathrm{r}} \theta^{\mathrm{i}} R_{\varphi \theta}+\theta^{\mathrm{r}} \boldsymbol{\varphi}^{\mathrm{i}} R_{\theta \varphi}+\theta^{\mathrm{r}} \theta^{\mathrm{i}} R_{\theta \theta}\right) \cdot \boldsymbol{E}^{\mathrm{i}}
$$

Let us choose $\mathbf{n}=\mathbf{z}$ and $\mathbf{a}_{t}=\mathbf{x}$. Then the reflection coefficients can be written in terms of the angles of incidence $\theta^{\mathrm{i}}$ and $\varphi^{\mathrm{i}}$ in Fig. 5, as

$$
\begin{gathered}
R_{\varphi \varphi}=R_{\theta \theta}=\left\{\begin{array}{lr}
\frac{(A)^{2}-(B)^{2}}{D}, & \text { oblique incidence } \\
\cos \left(2 \varphi^{\mathrm{i}}\right), & T_{s} \neq 0, \text { normal incidence }
\end{array}\right. \\
R_{\varphi \theta}=-R_{\theta \varphi}=\left\{\begin{array}{lr}
\frac{-2 A B}{D}, & \text { oblique incidence } \\
-\sin \left(2 \varphi^{\mathrm{i}}\right), & T_{s} \neq 0, \text { normal incidence }
\end{array}\right.
\end{gathered}
$$

with

$$
\begin{gathered}
A=T_{s} \cos \theta^{\mathrm{i}} \cos \varphi^{\mathrm{i}}, \quad B=T_{s} \sin \varphi^{\mathrm{i}}+T_{d} \sin \theta^{\mathrm{i}}, \\
D=\left(T_{d}+T_{s} \sin \theta^{\mathrm{i}} \sin \varphi^{\mathrm{i}}\right)^{2}+\left(T_{s}^{2}-T_{d}^{2}\right)\left(\cos \theta^{\mathrm{i}}\right)^{2} .
\end{gathered}
$$

Derivation of the reflection coefficients is presented in the Appendix.

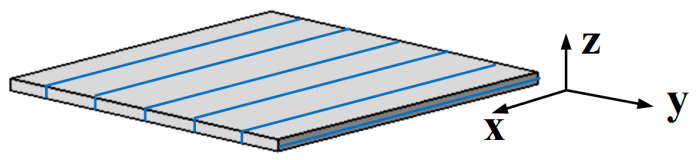

Fig. 6. An SHDB square plate with an edge length of $3 \lambda$ and thickness of $0.1 \lambda$. The blue lines indicate the direction of $\mathbf{a}_{t}$ on each face of the plate.

Since with the proposed SIE method it is not possible to compute scattering by an infinite SHDB surface, we consider a finite large square plate with an edge length of $3 \lambda$ and thickness of $0.1 \lambda$, as illustrated in Fig. 6 . The SHDB boundary condition is defined on the surface of the plate so that on the surfaces parallel to the $y-z$ plane, the direction of $\mathbf{a}_{t}$ is parallel to $z$ axis. On the other surfaces, vector $\mathbf{a}_{t}$ is parallel to the $x$ axis. The coordinate system is defined similarly as in Fig. 5.

We calculate the RCS of the plate under plane waves with different incident directions. The incident direction of the plane wave is the same as $\mathbf{u}^{\mathrm{i}}$ shown in Fig. 5. The direction of the incident electric field is $\theta^{\mathrm{i}}$ and the operating frequency is $300 \mathrm{MHz}$. The mesh size is $\lambda / 10$ and the number of edges on the triangular mesh is 6546 .

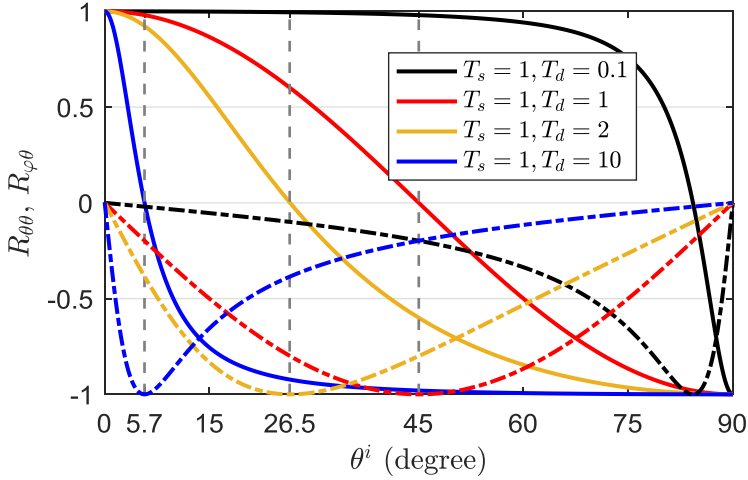

Fig. 7. The reflection dyadic components of an infinite plane for a plane wave incident at $\varphi^{\mathrm{i}}=0$ as functions of $\theta^{\mathrm{i}}$. Curves in the figure correspond to reflection coefficients $R_{\theta \theta}$ (solid line) and $R_{\varphi \theta}$ (dash-dot line).

1) Oblique incidence in $x$ - $z$ plane: First, we consider the scattering from the plate illuminated by an oblique incident plane wave propagating in $x-z$ plane. For the infinite plate, the values of the co- and cross-polarized components of the reflection dyadic with $\varphi^{\mathrm{i}}=0^{\circ}$ and as functions of $\theta^{\mathrm{i}}$ are plotted in Fig. 7 for four sets of parameter values $T_{s}=1, T_{d}=$ $(0.1,1,2,10)$. It is shown that the polarization of a plane wave reflected from the SHDB boundary with $T_{d}=1,2,10$ will be reversed when $\theta^{\mathrm{i}}=45^{\circ}, 26.5^{\circ}, 5.7^{\circ}$, respectively.

We next compute numerically the bistatic RCS in $x-z$ plane of the finite plate with parameter values $T_{s}=1, T_{d}=$ $(0.1,1,10)$ under the incident direction $\theta^{\mathrm{i}}=45^{\circ}, \varphi^{\mathrm{i}}=0^{\circ}$. The results obtained by NSIE are plotted in Fig. 8 and compared with the results of the PO method. It can be observed that for $T_{s}=T_{d}=1$, the cross-polarized RCS are much larger than the co-polarized RCS, while the co-polarized RCS are much larger than the cross-polarized RCS for $T_{s}=1, T_{d}=0.1$ and $T_{s}=1, T_{d}=10$. This is consistent with the results of Fig. 7 . The overall performance of the results obtained by the NSIE method is in line with the PO results. Fig. 9 shows the results of the corresponding computations for the incidence direction $\theta^{\mathrm{i}}=26.5^{\circ}, \varphi^{\mathrm{i}}=0^{\circ}$.

At last, we calculate the bistatic RCS in $x-z$ plane of the plate with parameter values $T_{s}=1, T_{d}=(0.1,10)$ under the incident direction $\theta^{\mathrm{i}}=5.7^{\circ}, \varphi^{\mathrm{i}}=0^{\circ}$. The results obtained by the NSIE method and the PO method are in good agreement for $T_{d}=0.1$, as shown in Fig. 10. However, the SIE results for $T_{d}=10$ disagree with the PO results, and the property to reverse the polarization of the plane wave is not observed. From the dependence of the reflection dyadic on the incidence angle $\theta^{\mathrm{i}}$ depicted in Fig. 7, we observe that the reflection dyadic for $T_{d}=10$ changes radically as $\theta^{\mathrm{i}}$ varies around $5.7^{\circ}$. In this case, a slight change of incident direction will have a great influence on the value of the scattered field. This is because the edge diffractions have a significant effect on the scattered field for the plate with finite size. As is well known, the accuracy of the PO approximation can be poor near sharp wedges due to the lack of edge diffraction.

2) Oblique incidence in $y$ - $z$ plane: Then, we consider the scattering from the plate illuminated by an oblique incident plane wave propagating in $y-z$ plane. For oblique incidence 


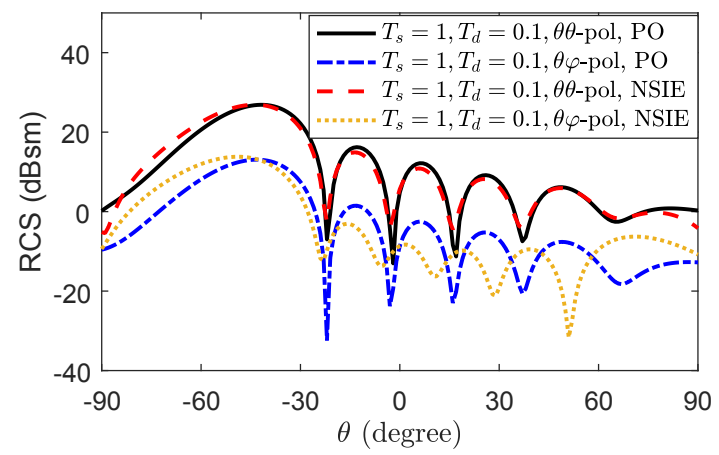

(a)

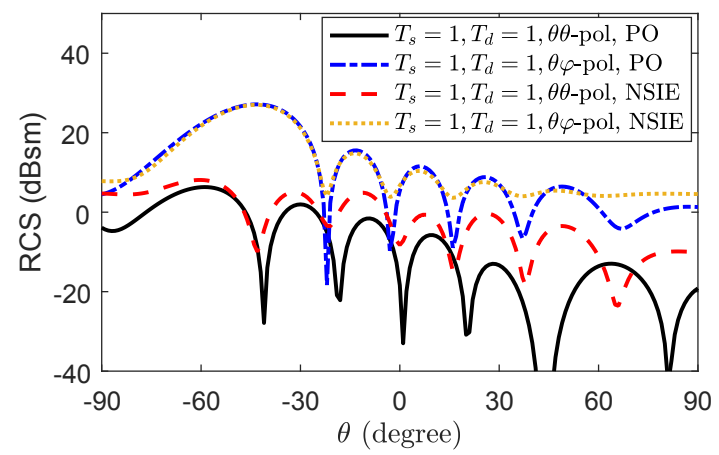

(b)

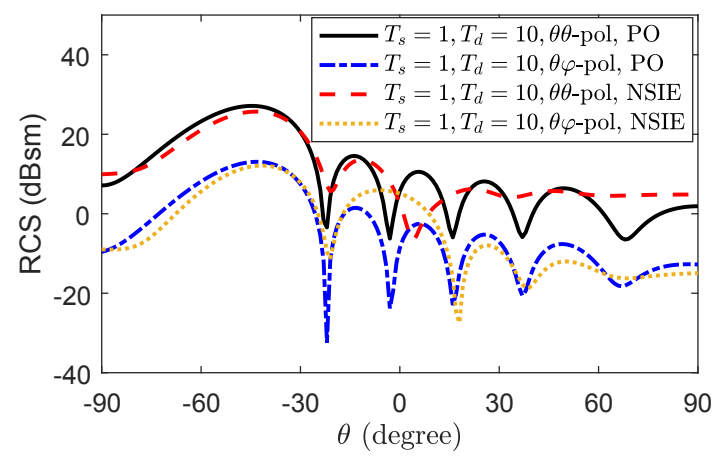

(c)

Fig. 8. Bistatic RCS of the plate in Fig. 6 illuminated by plane wave with $\theta^{\mathrm{i}}=45^{\circ}, \varphi^{\mathrm{i}}=0^{\circ}$ at a frequency of $300 \mathrm{MHz}$. The results are shown in $x-z$ plane. (a) $T_{s}=1, T_{d}=0.1$. (b) $T_{s}=1, T_{d}=1$. (c) $T_{s}=1, T_{d}=10$.

in $y$ - $z$ plane $\left(\varphi^{\mathrm{i}}=90^{\circ}\right), R_{\varphi \varphi}=R_{\theta \theta}=-1$ and $R_{\varphi \theta}=$ $R_{\theta \varphi}=0$ for all values of $\theta^{\mathrm{i}}, T_{s}$ and $T_{d}$, whence there is no cross polarization in the case of infinite plane. We calculate the bistatic RCS in $y-z$ plane of the finite plate with parameter values $T_{s}=1, T_{d}=(0.1,1,10)$ under the incident direction $\theta^{\mathrm{i}}=45^{\circ}, \varphi^{\mathrm{i}}=90^{\circ}$. The results obtained by NSIE and the PO method are plotted in Fig. 11. The value of the cross-polarized RCS is much smaller compared to the co-polarized one. The cross-polarized RCS of PO method is very small and is not shown in Fig. 11.

Except for the special case in Fig. 10 (b), we can also observe the specular reflection of the plate from the above results for oblique incidence. The largest value of RCS occurs at the point of $\theta=-\theta^{\mathrm{i}}$, which is the same as the reflection angle in the specular reflection.

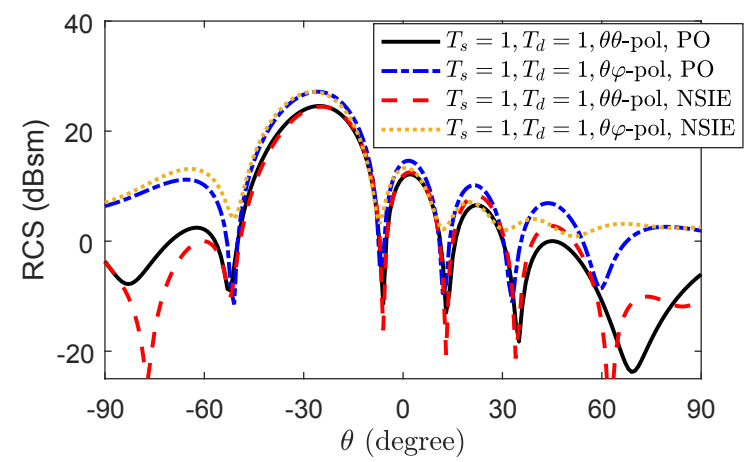

(a)

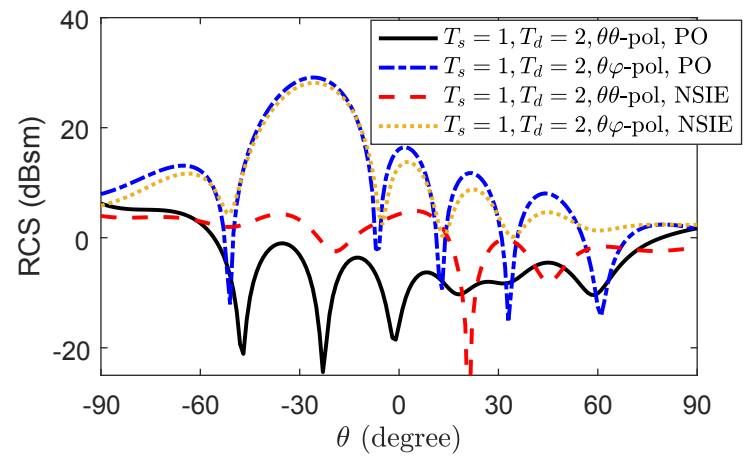

(b)

Fig. 9. Bistatic RCS of the plate in Fig. 6 illuminated by plane wave with $\theta^{\mathrm{i}}=26.5^{\circ}, \varphi^{\mathrm{i}}=0^{\circ}$ at a frequency of $300 \mathrm{MHz}$. The results are shown in $x-z$ plane. (a) $T_{s}=1, T_{d}=1$. (b) $T_{s}=1, T_{d}=2$.

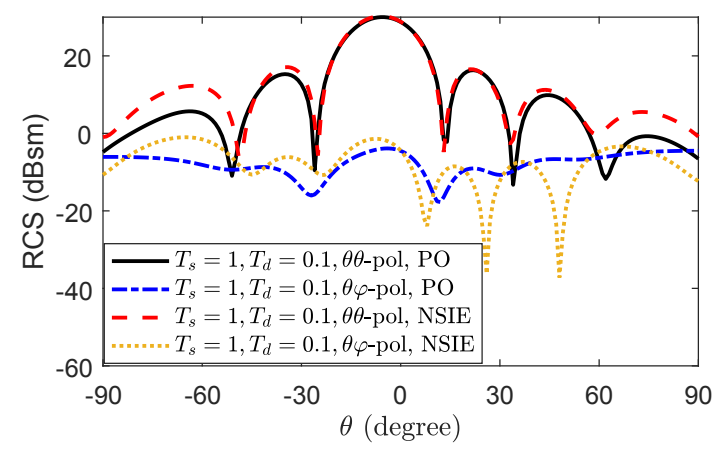

(a)

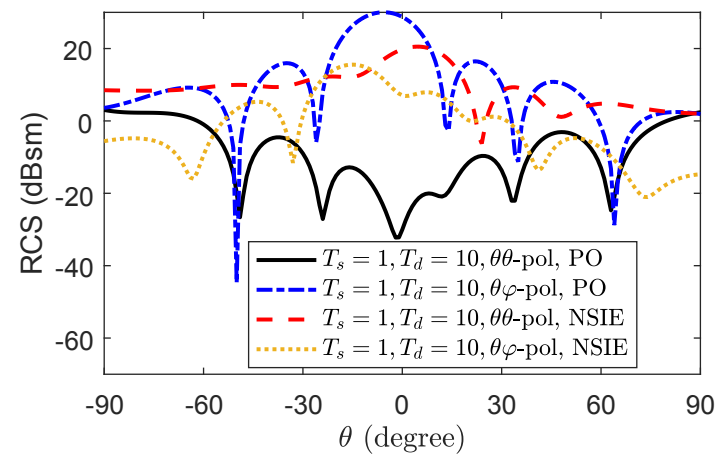

(b)

Fig. 10. Bistatic RCS of the plate in Fig. 6 illuminated by plane wave with $\theta^{\mathrm{i}}=5.7^{\circ}, \varphi^{\mathrm{i}}=0^{\circ}$ at a frequency of $300 \mathrm{MHz}$. The results are shown in $x-z$ plane. (a) $T_{s}=1, T_{d}=0.1$. (b) $T_{s}=1, T_{d}=10$. 


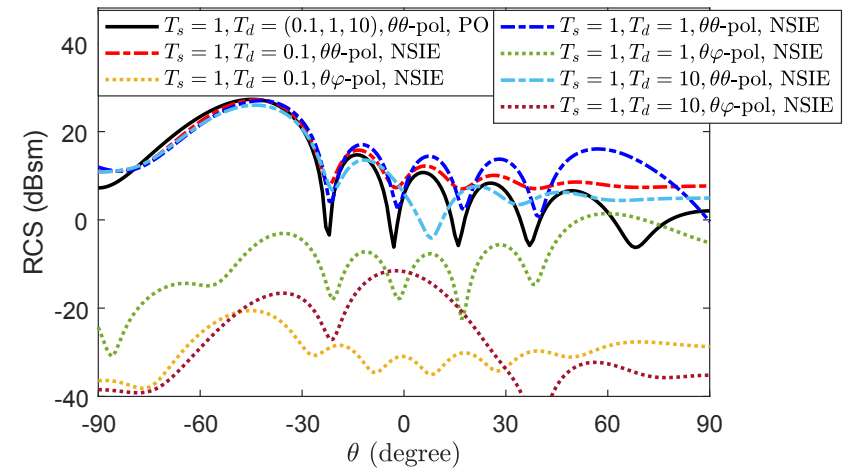

Fig. 11. Bistatic RCS of the plate in Fig. 6 illuminated by plane wave with $\theta^{\mathrm{i}}=45^{\circ}, \varphi^{\mathrm{i}}=90^{\circ}$ at a frequency of $300 \mathrm{MHz}$. The results are shown in $y-z$ plane.

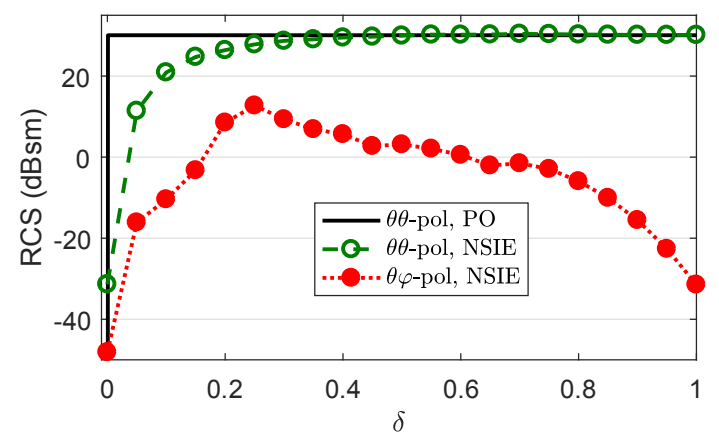

(a)

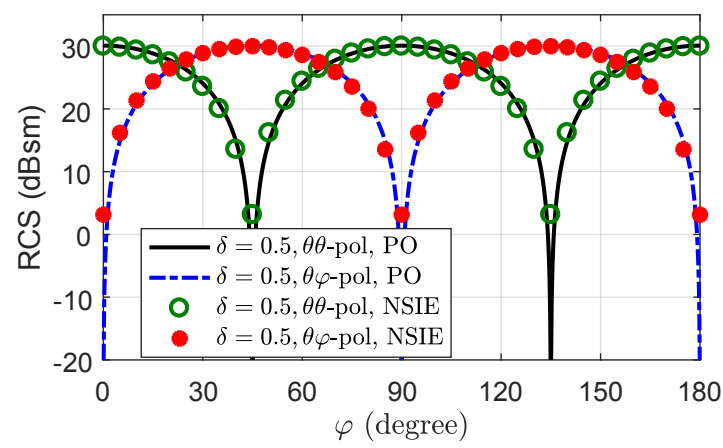

(b)

Fig. 12. Monostatic RCS of the plate in Fig. 6 illuminated by plane wave with normal incidence at a frequency of $300 \mathrm{MHz}$. (a) Monostatic RCS as a function of $\delta$ with $\varphi^{\mathrm{i}}=0^{\circ}$. (b) Monostatic RCS as a function of $\varphi^{\mathrm{i}}$ with $\delta=0.5$.

3) Normal incidence: As a last example, we consider the scattering from the plate illuminated by a plane wave with normal incidence. From Eqs. (34) and (35) we know that for normal incidence to an infinite plane, except for the case $T_{s}=$ 0 , the value of the reflection dyadic is the same for all values of $T_{s}$ and $T_{d}$. Thus, for normal incidence, the boundary acts as an $\mathrm{SH}$ boundary when $T_{s} \neq 0$ and suddenly changes to a DB boundary when $T_{s}=0$ and reflection vanishes.

Let us define $T_{d}=1-\delta$ and $T_{s}=\delta$ and calculate the monostatic RCS of the plate as $\delta$ is increased from 0 to 1 . The plate is illuminated by a normally incident plane wave $\left(\mathbf{u}^{\mathrm{i}}=-\mathbf{z}\right)$ with $\varphi^{\mathrm{i}}=0^{\circ}$. The results computed with NSIE and PO are plotted in Fig. 12 (a) as a function of $\delta$. The cross-

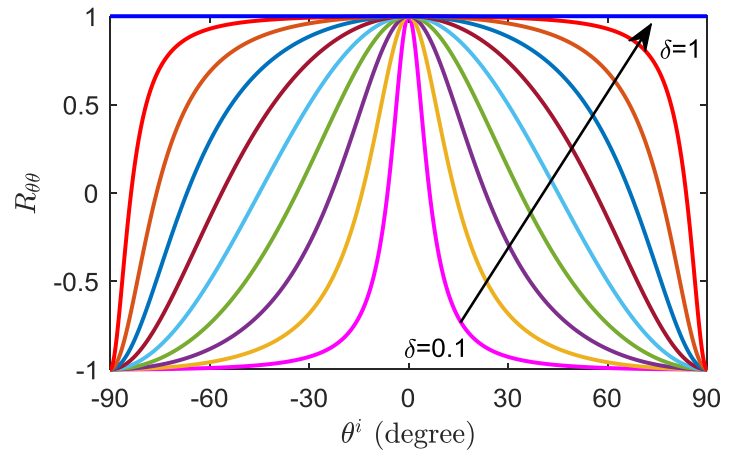

(a)

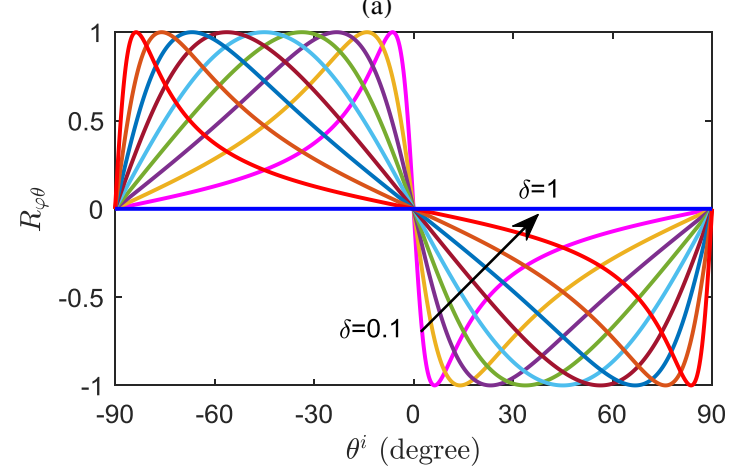

(b)

Fig. 13. Components of the reflection dyadic for a plane wave incident at $\varphi^{\mathrm{i}}=0^{\circ}$. (a) Reflection coefficient $R_{\theta \theta}$. (b) Reflection coefficient $R_{\varphi \theta}$.

polarized RCS of PO is very small and is not shown in Fig. 12 (a). As with the above analysis, the results of PO keep constant for $\delta \neq 0$ and suddenly become very small when $\delta=0$. The results of NSIE are generally consistent with the PO results. As $\delta$ decreases, the difference between the SIE and PO results increases. The cross-polarized scattering values of NSIE are low (more than $20 \mathrm{~dB}$ below co-polarization) and also due to the finite size of the plate which is non-symmetric according to Fig. 6. In order to analyze the results, we plot the value of the co- and cross-polarized reflection dyadic given in Eqs. (34) and (35) with $\varphi^{\mathrm{i}}=0^{\circ}$ as a function of $\theta^{\mathrm{i}}$ in Fig. 13 for various values of $\delta$. These results indicate that the smaller the value of $\delta$, the more drastic the change of the reflection dyadic, as $\theta^{\mathrm{i}}$ varies around $0^{\circ}$. In this particular case, the reflection of an infinite plate differs significantly from the reflection of a finite sized plate.

Changing the polarization of a normally incident field, the monostatic RCS of the plate with $\delta=0.5$ is calculated and drawn in Fig. 12 (b) as a function of $\varphi^{\mathrm{i}}$. The results of NSIE and PO agree well with each other. From Eqs. (34) and (35), we can observe that the co-polarized response has $\cos \left(2 \varphi^{\mathrm{i}}\right)$ dependence and the cross-polarized one has $\sin \left(2 \varphi^{\mathrm{i}}\right)$ dependence, which are consistent with our results in Fig. 12 (b).

\section{CONCLUSION}

A surface integral equation (SIE) method is developed for electromagnetic scattering by arbitrarily shaped 3-D objects with Soft-and-Hard/DB (SHDB) boundary condition. In the proposed method, the SHDB boundary condition is expressed 
in a vector form, which can be combined with the field integral equations to constitute the SIE formulation that can be discretized using MoM with standard basis functions, such as RWG functions. Different combinations of the boundary conditions and integral equations are investigated. Numerical experiments show that the proposed non-square integral equation (NSIE) formulation can provide accurate and stable solutions for SHDB surfaces with different boundary condition parameters. The reflection properties of the SHDB boundary, such as the changes of polarization at specific angles of incidence, are observed by the NSIE method and verified with the PO approximation.

The proposed method can be applied for any linear boundary condition that can be expressed in terms of the surface currents. The particularly attractive feature of the method is that any special numerical methods or basis functions are not needed. The implementation is based on the standard GalerkinRWG approach.

\section{APPENDIX A}

\section{DERIVATION OF THE REFLECTION COEFFICIENTS}

In this Appendix, we present the derivation of the reflection coefficients $R_{\varphi \varphi}, R_{\theta \theta}, R_{\varphi \theta}$ and $R_{\theta \varphi}$ given in Eqs. (34) and (35).

Let us define the unit dyadics $\overline{\bar{I}}_{i}=\boldsymbol{\varphi}^{\mathrm{i}} \boldsymbol{\varphi}^{\mathrm{i}}+\boldsymbol{\theta}^{\mathrm{i}} \boldsymbol{\theta}^{\mathrm{i}}+\mathbf{u}^{\mathrm{i}} \mathbf{u}^{\mathrm{i}}$ and $\overline{\bar{I}}_{r}=\boldsymbol{\varphi}^{\mathrm{r}} \boldsymbol{\varphi}^{\mathrm{r}}+\boldsymbol{\theta}^{\mathrm{r}} \boldsymbol{\theta}^{\mathrm{r}}+\mathbf{u}^{\mathrm{r}} \mathbf{u}^{\mathrm{r}}$. Then by applying the relations $\mathbf{u}^{\mathrm{r}} \cdot \boldsymbol{E}^{\mathrm{r}}=0$ and $\mathbf{u}^{\mathrm{i}} \cdot \boldsymbol{E}^{\mathrm{i}}=0$, the relations of the incident and reflected fields in (4) can be written as

$$
\begin{aligned}
& \boldsymbol{E}^{\mathrm{r}}=\overline{\bar{I}}_{r} \cdot \overline{\bar{R}}_{E} \cdot \overline{\bar{I}}_{i} \cdot \boldsymbol{E}^{\mathrm{i}} \\
& =\left(\boldsymbol{\varphi}^{\mathrm{r}} \boldsymbol{\varphi}^{\mathrm{i}} R_{\varphi \varphi}+\varphi^{\mathrm{r}} \theta^{\mathrm{i}} R_{\varphi \theta}+\theta^{\mathrm{r}} \boldsymbol{\varphi}^{\mathrm{i}} R_{\theta \varphi}+\theta^{\mathrm{r}} \theta^{\mathrm{i}} R_{\theta \theta}\right) \cdot \boldsymbol{E}^{\mathrm{i}} .
\end{aligned}
$$

Here the reflection coefficients

$$
\begin{aligned}
R_{\varphi \varphi} & =\varphi^{\mathrm{r}} \cdot \overline{\bar{R}}_{E} \cdot \boldsymbol{\varphi}^{\mathrm{i}}, & R_{\varphi \theta}=\boldsymbol{\varphi}^{\mathrm{r}} \cdot \overline{\bar{R}}_{E} \cdot \theta^{\mathrm{i}} \\
R_{\theta \varphi} & =\theta^{\mathrm{r}} \cdot \overline{\bar{R}}_{E} \cdot \boldsymbol{\varphi}^{\mathrm{i}}, & R_{\theta \theta}=\theta^{\mathrm{r}} \cdot \overline{\bar{R}}_{E} \cdot \theta^{\mathrm{i}} .
\end{aligned}
$$

Let $\mathbf{u}^{\mathrm{i}}=u_{1} \mathbf{x}+u_{2} \mathbf{y}+u_{3} \mathbf{z}$ and $\boldsymbol{\varphi}^{\mathrm{i}}=\varphi_{1} \mathbf{x}+\varphi_{2} \mathbf{y}+\varphi_{3} \mathbf{z}$. Substituting Eqs. (5), (31), (32), and $\mathbf{n}=\mathbf{z}, \mathbf{a}_{t}=\mathbf{x}$ to Eq. (38), the reflection coefficients for oblique incidence simplifies to

$$
\begin{gathered}
R_{\varphi \varphi}=R_{\theta \theta}=\frac{T_{s}^{2} u_{3}^{2} u_{1}^{2}-\left[T_{d}\left(1-u_{3}^{2}\right)-T_{s} u_{2}\right]^{2}}{\left(u_{1}^{2}+u_{2}^{2}\right)\left[\left(T_{d}-T_{s} u_{2}\right)^{2}+u_{3}^{2}\left(T_{s}^{2}-T_{d}^{2}\right)\right]} \\
R_{\varphi \theta}=-R_{\theta \varphi}=\frac{-2 T_{s} u_{3} u_{1}\left[T_{d}\left(1-u_{3}^{2}\right)-T_{s} u_{2}\right]}{\left(u_{1}^{2}+u_{2}^{2}\right)\left[\left(T_{d}-T_{s} u_{2}\right)^{2}+u_{3}^{2}\left(T_{s}^{2}-T_{d}^{2}\right)\right]} .
\end{gathered}
$$

For normal incidence, substituting Eqs. (5), (30), (32), and $\mathbf{n}=\mathbf{z}, \mathbf{a}_{t}=\mathbf{x}$ to (38), the reflection coefficients can be expressed as

$$
R_{\varphi \varphi}=R_{\theta \theta}=\varphi_{2}^{2}-\varphi_{1}^{2}, \quad R_{\varphi \theta}=-R_{\theta \varphi}=2 \varphi_{2} \varphi_{1}
$$

for $T_{s} \neq 0$. When $T_{s}=0$, the boundary is the DB boundary and there is no reflection from the boundary for normally incident plane wave.

\section{REFERENCES}

[1] I. V. Lindell and A. Sihvola, Boundary Conditions in Electromagnetics. Wiley-IEEE Press, 2020.

[2] D. J. Hoppe and Y. Rahmat-Samii, Impedance Boundary Conditions in Electromagnetics. Washington, DC, USA: Taylor \& Francis, 1995.

[3] E. Lier, "Review of soft and hard horn antennas, including metamaterialbased hybrid-mode horns," IEEE Antennas Propag. Magaz., vol. 52, no. 2, pp. 31-39, Apr. 2010.

[4] P. Kildal, "Fundamental properties of canonical soft and hard surfaces, perfect magnetic conductors and the newly introduced DB surface and their relation to different practical applications including cloaking," in Proc. ICEAA, Torino, Italy, Aug. 2009, pp. 607-610.

[5] B. Zhang, H. Chen, B.-I. Wu, and J. A. Kong, "Extraordinary surface voltage effect in the invisibility cloak with an active device inside," Phys. Rev. Lett., vol. 100, no. 6, Feb. 2008.

[6] P.-S. Kildal, "Definition of artificially soft and hard surfaces for electromagnetic waves," Electron. Lett., vol. 24, no. 3, pp. 168-170, 1988.

[7] V. Rumsey, "Some new forms of Huygens' principle," IRE Trans. Antennas Propag., vol. 7, no. 5, pp. 103-116, Dec. 1959.

[8] D. Zaluški, S. Hrabar, and D. Muha, "Practical realization of DB metasurface," Appl. Phys. Lett., vol. 104, no. 23, p. 234106, Jun. 2014.

[9] I. V. Lindell and A. Sihvola, "Electromagnetic wave reflection from boundaries defined by general linear and local conditions," IEEE Trans. Antennas Propag., vol. 65, no. 9, pp. 4656-4663, Sep. 2017.

[10] — , "Soft-and-hard/DB boundary conditions defined by a skewonaxion medium," IEEE Trans. Antennas Propag., vol. 61, no. 2, pp. 768 774, Feb. 2013

[11] I. V. Lindell and A. Sihvola, "Surface waves on SHDB boundary," IEEE Antennas Wireless Propag. Lett., vol. 13, pp. 1027-1030, 2014.

[12] - "Generalized soft-and-hard/DB boundary," IEEE Transactions on Antennas and Propagation, vol. 65, no. 1, pp. 226-233, 2017.

[13] S. Rao, D. Wilton, and A. Glisson, "Electromagnetic scattering by surfaces of arbitrary shape," IEEE Trans. Antennas Propag., vol. 30, no. 3, pp. 409-418, May. 1982.

[14] W. Gordon, "Far-field approximations to the Kirchoff-Helmholtz representations of scattered fields," IEEE Trans. Antennas Propag., vol. 23, no. 4, pp. 590-592, Jul. 1975.

[15] P. Ylä-Oijala, M. Taskinen, and S. Järvenpää, "Surface integral equation formulations for solving electromagnetic scattering problems with iterative methods," Radio Sci., vol. 40, 2005.

[16] B. Kong, P. Ylä-Oijala, and A. Sihvola, "Surface integral equation method for generalized soft-and-hard boundary condition," IEEE Trans. Antennas Propag., vol. 68, no. 5, pp. 3807-3814, May. 2020.

[17] S. Yan and J.-M. Jin, "Self-dual integral equations for electromagnetic scattering from IBC objects," IEEE Trans. Antennas Propag., vol. 61, no. 11, pp. 5533-5546, Nov. 2013.

[18] P. Ylä-Oijala, S. P. Kiminki, H. Wallén, and A. Sihvola, "Uniform surface integral equation formulation for mixed impedance boundary conditions," IEEE Trans. Antennas Propag., vol. 63, no. 12, pp. 57185726, Dec. 2015

[19] P. Ylä-Oijala and S. Järvenpää, "New trends in frequency-domain surface integral equations," in New Trends in Computational Electromagnetics, Ö. Ergül, Ed. London: Scitech Publishing, Jan. 2019, ch. 3.

[20] S. Yan, J. Jin, and Z. Nie, "Analysis of electrically large problems using the augmented efie with a calderón preconditioner," IEEE Trans. Antennas Propag., vol. 59, no. 6, pp. 2303-2314, 2011.

[21] J. Markkanen, P. Ylä-Oijala, and A. Sihvola, "Computation of scattering by DB objects with surface integral equation method," IEEE Trans. Antennas Propag., vol. 59, no. 1, pp. 154-161, Jan. 2011. 